\title{
Rational Modeling Algorithm for Passive Microwave Structures and Systems
}

Dirk Deschrijver, Tom Dhaene, Oliver Salazar Celis and Annie Cuyt

\begin{abstract}
An efficient identification method is proposed for passive rational approximation of frequency domain responses. The method is applied to compute a transfer function from tabulated S-parameter data of a multiport microwave filter. Numerical results validate the robustness and efficacy of the modeling approach.
\end{abstract}

\section{Introduction}

Broadband characterization of passive linear systems, based on measured and simulated data has become a topic of intense research, mainly due to the increasing speed and decreasing size of electronic circuits. While the operating frequency is increasing well above the multi-GHz frequency range, parasitic effects that were previously ignored cannot be overlooked anymore for accurate system level analysis [1]. This complicates the modeling and design of RF, microwave and millimeter-wave components and systems. The number of design failures caused by signal and power integrity problems has become problematic because the existing design tools and modeling methodologies cannot address these issues in an appropriate way [2,3].

Common approaches to tackle these problems are based on the approximation of tabulated S-parameter frequency responses using rational functions and subsequently synthesizing a SPICE compatible macromodel $[4,5]$. These macromodels approximate the complex electromagnetic (EM) behavior of high-speed multi-port systems at the input-output ports in the frequency domain by rational functions [6].

Dirk Deschrijver, Tom Dhaene

Ghent University - IBBT, Sint Pietersnieuwstraat 41, 9000 Ghent, Belgium, e-mail: \{dirk.deschrijver,tom.dhaene\}@intec.ugent.be

Oliver Salazar Celis, Annie Cuyt

University of Antwerp, Middelheimlaan 1, 2020 Antwerp, Belgium, e-mail: \{oliver.salazarcelis,annie.cuyt $\} @$ ua.ac.be 
The advantage of this approach is that a circuit can be modeled as a black-box, and that no knowledge of its internal logic is required for the modeling process [7].

In 1999, the iterative Vector Fitting (VF) technique was introduced [8-10] to compute such macromodels in a robust and efficient way. It is applicable to both smooth and resonant responses with high orders and wide frequency bands. An additional advantage is that stability of the poles is easily enforced by a simple poleflipping scheme. Since VF is now adopted in many societies of applied engineering, including power systems and microwave systems, it has drawn a lot of attention from researchers, and it has become the de-facto standard for rational approximation. A general overview of the VF methodology was recently presented in [11].

A known restriction of the technique is that the computed macromodels are not guaranteed to be passive by construction. Nevertheless, passivity of the macromodel is of crucial importance since a non-passive macromodel may lead to unstable transient simulations in an unpredictable manner $[12,13]$. This paper applies a new passivity enforcement technique that is able to enforce passivity to a non-passive rational macromodel by means of an overdetermined least-squares fitting algorithm [14].

Numerical results show that the presented approach achieves an excellent tradeoff between computation time and accuracy preservation of the macromodel.

\section{Vector Fitting Algorithm}

Given a set of S-parameter data samples $\left\{s_{k}, H_{m n}\left(s_{k}\right)\right\}_{k=1}^{K}$, the VF algorithm [8] computes a rational macromodel that matches the frequency response by solving several least squares problems in successive iteration steps $(t=0, \ldots, T)$, where $1 \leq$ $m, n \leq Q$ denotes the $m^{\text {th }}$ row and the $n^{\text {th }}$ column of a $Q$-port transfer matrix $H$.

$$
\arg \min \left|\left(\Psi H_{m n}\right)^{t}(s)-\Psi^{t}(s) H_{m n}(s)\right|^{2}
$$

Both $\left(\Psi H_{m n}\right)^{t}(s)$ and $\Psi^{t}(s)$ in (1) are expanded as a linear combination of partial fractions (or orthonormal rational functions [15]) that share common poles $\left\{a_{p}^{t}\right\}_{p=1}^{P}$.

$$
\begin{aligned}
\left(\Psi H_{m n}\right)^{t}(s) & =\sum_{p=1}^{P} \frac{c_{p, m n}^{t}}{s-a_{p}^{t}}+c_{0, m n}^{t} \\
\Psi^{t}(s) & =\sum_{p=1}^{P} \frac{\tilde{c}_{p}^{t}}{s-a_{p}^{t}}+\tilde{c}_{0}^{t}
\end{aligned}
$$

In the first iteration step $(t=0)$, the initial starting poles $a_{p}^{0}$ are selected according to a heuristical scheme [8]. It is trivial to transform (1) into an overdetermined set of least-squares equations $A^{t} x^{t}=b^{t}$, where the solution vector $x^{t}$ contains the unknown coefficients $\left\{c_{p, m n}^{t}, \tilde{c}_{p}^{t}\right\}_{p=0}^{P}$. Based on the coefficients $\left\{\tilde{c}_{p}^{t}\right\}$, it is possible to obtain a new set of relocated poles by solving the zeros of $\Psi^{t}(s)$ as an eigenvalue problem. These relocated poles replace the initial set of poles, and the process of solving (1) 
is repeated iteratively until the poles are converged to some quasi-optimal position. The trivial null solution of (1) is avoided by setting one coefficient $\tilde{c}_{0}^{t}=1$ or by adding an additional relaxation constraint to (1), as in [9]. It was shown in a previous report that this process is related to the Sanathanan-Koerner iteration [16].

In the final iteration step $(t=T)$, the transfer function $\left(\Psi H_{m n}\right)^{T}(s)$ in partial fraction form is obtained by solving (1) with $\Psi^{T}(s)=1$. All the details about this Vector Fitting procedure are extensively reported in literature, see [8, 17].

\section{Passivity Enforcement Algorithm}

The passivity constraints for stable and causal macromodels in the scattering case require that the singular values of the transfer matrix $H$ are unitary bounded $[18,19]$

$$
I-H_{m n}^{H}(j \omega) H_{m n}(j \omega) \geq 0 \quad \forall \omega \in \mathbb{R}
$$

By computing the eigenvalues of an associated Hamiltonian matrix, it is possible to assess algebraically the passivity of the model [20,21]. In addition, the eigenvalues can be used to exactly pinpoint the boundaries of possible passivity violations. To compensate any occuring violations, the following procedure is used. First a dense set of frequencies $\omega_{k}$ is used to evaluate the singular value decomposition

$$
H_{m n}\left(j \omega_{k}\right)=U_{k} \Sigma_{k} V_{k}^{H}
$$

where $\Sigma_{k}=\operatorname{diag}\left(\sigma_{1, k}, \ldots, \sigma_{Q, k}\right)$ is a diagonal matrix that contains the singular values on its main diagonal. The set contains all the samples of the original frequency response, combined with some additional samples in the vicinity of passivity violations [22]. These samples are included to ensure a good resolution of the singular value trajectories. Then, a set of violation parameters is constructed as follows

$$
\Delta H_{m n}\left(j \omega_{k}\right)=U_{k} \Delta \Sigma_{k} V_{k}^{H}
$$

with $\Delta \Sigma_{k}=\operatorname{diag}\left(\Delta \sigma_{1, k}, \ldots, \Delta \sigma_{Q, k}\right)$, where

$$
\begin{array}{r}
\Delta \sigma_{q, k}=0 \text { if } \sigma_{q, k} \leq \gamma \\
\Delta \sigma_{q, k}=\sigma_{q, k}-\gamma \text { if } \sigma_{q, k}>\gamma
\end{array}
$$

The threshold parameter $\gamma$ is chosen close to, but less than 1. In general, larger values of $\gamma$ (closer to 1 ) are able to better preserve the accuracy of the model, but may require additional iterations to achieve passivity. In order to make the model passive, a new set of offset residues $\Delta c_{p, m n}^{T}$ is computed by fitting the violation parameters $\Delta H_{m n}\left(j \omega_{k}\right)$ using the same set of poles of the original model.

$$
\arg \min \left|\left(\Psi H_{m n}\right)^{T}(j \omega)-\Delta H_{m n}(j \omega)\right|^{2}
$$




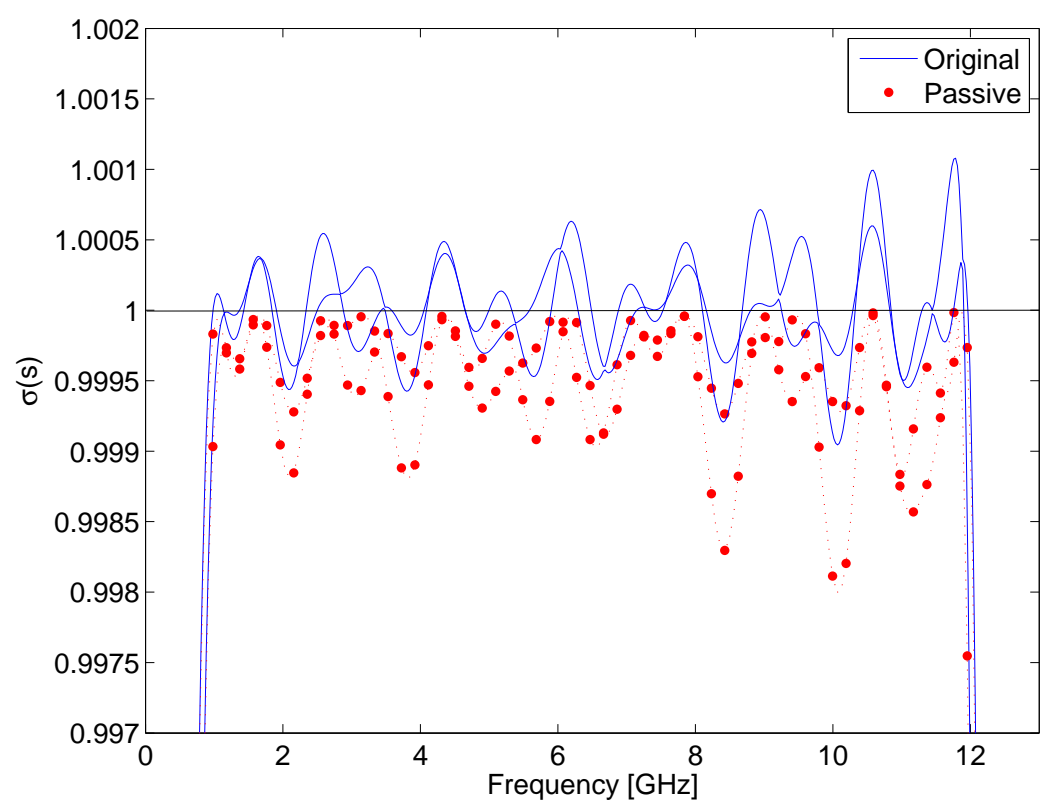

Fig. 1 Quarter Wavelength : Singular values of scattering matrix.

A new model with reduced passivity violations is obtained by substituting $c_{p, m n}^{T}$ with $c_{p, m n}^{T}-\Delta c_{p, m n}^{T}$. The process is repeated iteratively until passivity is reached.

\section{Example : Quarter Wavelength Filter}

In this example, the presented approach is used to compute a passive macromodel of a 2-port quarter wavelength filter. The scattering parameters of the structure are simulated in the frequency domain with a planar full-wave electromagnetic simulator over the frequency range $1 \mathrm{GHz}$ to $12 \mathrm{GHz}$. Then, the vector fitting algorithm is used to approximate the response by a 28 -pole strictly proper transfer function using 1000 data samples. The desired model accuracy of the S-parameters is $-60 \mathrm{~dB}$ or better, which corresponds approximately to 3 significant digits.

Even though the simulated data samples are passive, it is seen from Figure 1 that the resulting macromodel has several small in-band passivity violations. Therefore, the proposed passivity enforcement procedure is applied to compensate them, and the algorithm converges to a passive macromodel in a few iteration steps. It is found that the maximum singular value of the scattering matrix decreases monotonically in each iteration step of the algorithm. Figure 2 shows the magnitude of the original frequency response, and the deviation that is caused by the passivity perturbation. It is clear that the overall accuracy of the macromodel is well preserved. 


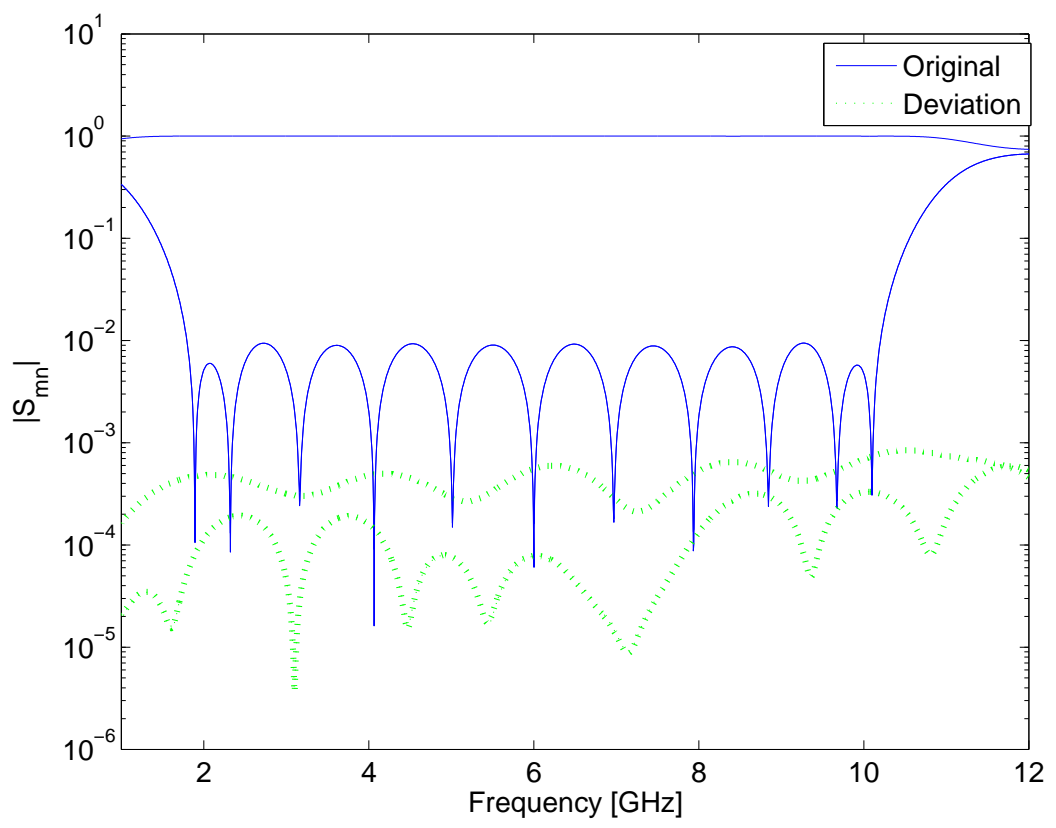

Fig. 2 Quarter Wavelength : Magnitude of matrix elements.

\section{Conclusions}

An efficient algorithm for passive macromodeling of microwave components is proposed. First, a robust macromodeling technique is used to approximate the frequency response with a rational transfer function. Then, the passivity of the model is assessed by using an algebraic passivity test. If the model is non-passive, an iterative passivity enforcement scheme is applied to perturb the model coefficients using a standard least-squares procedure. Numerical results confirm that the approach is effective, and that the perturbation does not compromise the accuracy of the model.

Acknowledgements This work was supported by the Fund for Scientific Research Flanders (FWO-Vlaanderen). Dirk Deschrijver is a post-doctoral research fellow of FWO-Vlaanderen.

\section{References}

1. S.-H. Min, "Automated Construction of Macromodels from Frequency Data for Simulation of Distributed Interconnect Networks", PhD thesis, Georgia Institute of Technology, USA, 2004.

2. K.-L. Choi, "Embedded Passives using Rational Functions in Multi-Layered Substrates", PhD thesis, Georgia Institute of Technology, USA, 1999.

3. E.P. Li, X.C. Wei, A.C. Cangellaris, E.X. Liu, Y.J. Zhang, M. D’Amore, J. Kim, T. Sudo, "Progress Review of Electromagnetic Compatibility Analysis Technologies for Packages, 
Printed Circuit Boards, and Novel Interconnects", IEEE Transactions on Electromagnetic Compatibility, vol. 52, no. 2, pp. 248-265, 2010.

4. D. Saraswat, R. Achar and M. Nakhla, "Enforcing Passivity for Rational Function Based Macromodels of Tabulated Data ", IEEE Conference on Electrical Performance of Electronic Packaging (EPEP 2003), Princeton, NJ (USA), pp. 295-298, 2003.

5. S. Lefteriu, A.C. Antoulas, "A New Approach to Modeling Multiport Systems from Frequency-Domain Data", IEEE Transactions on Computer-Aided Design of Integrated Circuits and Systems, vol. 29, no. 1, pp. 14-27, Jan. 2010.

6. A. Cuyt, "Nonlinear numerical methods and rational approximation", D. Reidel Publishing Company, Dordrecht (Holland), 1988.

7. B. Mutnury, "Macromodeling of Nonlinear Driver and Receiver Circuits ", PhD thesis, Georgia Institute of Technology, Georgia (USA), 2005.

8. B. Gustavsen and A. Semlyen, "Rational Approximation of Frequency Domain Responses by Vector Fitting", IEEE Transactions on Power Delivery, vol. 14, no. 3, pp. 1052-1061, 1999.

9. B. Gustavsen, "Improving the Pole Relocating Properties of Vector Fitting", IEEE Transactions on Power Delivery, vol. 21, no. 3, pp. 1587-1592, 2006.

10. D. Deschrijver, M. Mrozowski, T. Dhaene and D. De Zutter, "Macromodeling of Multiport Systems using a Fast Implementation of the Vector Fitting Method", IEEE Microwave and Wireless Components Letters, vol. 18, no. 6, pp. 383-385, 2008.

11. D. Deschrijver, B. Gustavsen and T. Dhaene, "Advancements in Iterative Methods for Rational Approximation in the Frequency Domain", IEEE Transactions on Power Delivery, vol. 22, no. 3, pp. 1633-1642, 2007.

12. D. Saraswat, R. Achar, M.S. Nakhla, "Global Passivity Enforcement Algorithm for Macromodels of Interconnect Subnetworks Characterized by Tabulated Data", IEEE Transactions on Very Large Scale Integration (VLSI) Systems, vol. 13, no. 7, pp. 819-832, Jul. 2005.

13. S. Grivet-Talocia, A. Ubolli, "A Comparative Study of Passivity Enforcement Schemes for Linear Lumped Macromodels", IEEE Transactions on Advanced Packaging, vol. 31, no. 4, pp. 673-683, Nov. 2008.

14. T. Dhaene, D. Deschrijver, N. Stevens, "Efficient Algorithm for Passivity Enforcement of Sparameter Based Macromodels", IEEE Transactions on Microwave Theory and Techniques, vol. 57, no. 2, pp. 415-420, 2009.

15. D. Deschrijver, B. Haegeman and T. Dhaene, "Orthonormal Vector Fitting: a Robust Macromodeling Tool for Rational Approximation of Frequency Domain Responses", IEEE Transactions on Advanced Packaging, vol. 30, no. 2, pp. 216-225, 2007.

16. W. Hendrickx, D. Deschrijver and T. Dhaene, "Some Remarks on the Vector Fitting Iteration", Post-Conference Proceedings of EMCI 2004, Mathematics in Industry, Springer-Verlag, pp. 134-138, 2006.

17. D. Deschrijver and T. Dhaene, "A Note on the Multiplicity of Poles in the Vector Fitting Macromodeling Method", IEEE Transactions on Microwave Theory and Techniques, vol. 55, no. 4, pp. 736-741, 2007.

18. S. Grivet-Talocia, "Passivity enforcement via perturbation of Hamiltonian matrices", IEEE Transactions on Circuits and Systems I, vol. 51, no. 9, pp. 17551769, Sep. 2004.

19. D. Deschrijver, T. Dhaene, "Fast Passivity Enforcement of S-parameter Macromodels by Pole Perturbation", IEEE Transactions on Microwave Theory and Techniques, vol. 57, no. 3, pp. 620-626, 2009.

20. S. Boyd, V. Balakrishnan and P. Kabamba, "A Bisection Method for Computing the $H_{\text {inf }}$ Norm of a Transfer Matrix and Related Problems", Mathematics of Control, Signals and Systems, vol. 2, pp. 207-219, 1989.

21. Z. Zhang and N. Wong, "Passivity Check of S-parameter Descriptor Systems via S-parameter Generalized Hamiltonian Methods", IEEE Transactions on Advanced Packaging, vol. 33, no. 4, pp. 1034-1042, Nov 2010.

22. A. Chinea, S. Grivet-Talocia, D. Deschrijver, T. Dhaene, L. Knockaert, "On the Construction of Guaranteed Passive Macromodels for High-Speed Channels", Design, Automation and Test in Europe (DATE 2010), Dresden (Germany), pp. 1142-1147, March 2010. 